\title{
Mechanism of praziquantel action at a parasitic flatworm ion channel
}

Sang-Kyu Park ${ }^{1}$, Lukas Friedrich ${ }^{2 \dagger}$, Nawal A. Yahya ${ }^{1,3}$, Claudia Rohr $^{1}$, Evgeny G. Chulkov ${ }^{1}$,

David Maillard ${ }^{4}$, Friedrich Rippmann², Thomas Spangenberg ${ }^{5 *}$, Jonathan S. Marchant ${ }^{1 *}$

${ }^{1}$ Department of Cell Biology, Neurobiology and Anatomy, Medical College of Wisconsin, Milwaukee WI 53226, USA.

${ }^{2}$ Computational Chemistry \& Biology, Global Research \& Development, Discovery Technologies, Merck Healthcare, Frankfurter Str. 250, 64293 Darmstadt, Germany.

${ }^{3}$ Department of Pharmacology, University of Minnesota Medical School, 312 Church St., Minneapolis, Minnesota 55455, USA.

${ }^{4}$ Central Process Development - Downstream Processing Services, Merck Performance Materials, Frankfurter Str. 250, 64293 Darmstadt, Germany.

${ }^{5}$ Global Health Institute of Merck, Ares Trading S.A., a subsidiary of Merck KGaA, Darmstadt, Germany, 1262 Eysins, Switzerland.

$\dagger$ These authors contributed equally to this work.

*Co-Corresponding authors:

Email: thomas.spangenberg@merckgroup.com (T.S.); JMarchant@mcw.edu (J.S.M.)

Keywords: schistosomiasis, calcium, TRP channel, trematodes, neglected tropical disease

Supplementary Material: Materials and Methods, Figures S1-6, Tables S1-6, Movie S1. 
Praziquantel (PZQ) is an essential medicine for treating parasitic flatworm infections such as schistosomiasis, which afflicts over $\mathbf{2 5 0}$ million people. However, PZQ is not universally effective, lacking activity against the liver fluke Fasciola. The reason for this insensitivity is unclear, as the mechanism of PZQ action is unknown. Here, we show PZQ activates a transient receptor potential melastatin ion channel (TRPMPza) in schistosomes by engaging a hydrophobic ligand binding pocket within the voltage-sensor like domain to cause $\mathrm{Ca}^{2+}$ entry and worm paralysis. PZQ activates TRPMPZQ homologues in other PZQsensitive flukes, but not Fasciola. However, a single amino acid change in the Fasciola TRPMPZQ binding pocket, to mimic schistosome TRPMPZQ, confers PZQ sensitivity. After decades of clinical use, the basis of PZQ action at a druggable TRP channel is resolved.

Praziquantel (PZQ) is an essential medicine for treating diseases caused by parasitic flatworms (helminths). PZQ is the key therapy for schistosomiasis (Bilharzia), a disease of poverty that afflicts $\sim 250$ million people worldwide $(1,2)$. The use and continued efficacy of PZQ is integral to the WHO roadmap of interventions to control schistosomiasis morbidity and to eliminate schistosomiasis as a public health problem by $2030(3,4)$. WHO estimates that at least 290 million people required preventive treatment in 2018. PZQ is also employed to treat other fluke and cestode infections that cause human disease, but is ineffective against the common liver fluke Fasciola. Fasciola is a zoonotic pathogen that causes clinical and veterinary disease of livestock, responsible for considerable economic damage (5). The explanation for the varied effectiveness of PZQ against blood and liver flukes is unclear, as the mechanism of action of PZQ is unknown.

$\mathrm{PZQ}$ is administered as a racemic mixture with the $(R)-\mathrm{PZQ}$ enantiomer causing a rapid contraction of adult schistosome worms and damage to the worm surface while the (S)-PZQ enantiomer is considerably less effective (6-9). Recently, we identified a schistosome transient receptor potential (TRP) channel that is activated by PZQ, although like many previously proposed PZQ targets $(10,11)$, a direct binding site for PZQ on the target remains undefined (12). TRP channels are polymodal ion 
channels frequently employed in sensory signaling roles, responding to ligands and environmental cues. The Schistosoma mansoni TRP channel activated by PZQ (Sm.TRPMPzQ; Smp_246790.5, chromosome 3) belongs to the TRP melastatin subfamily, which in vertebrates contain members responsive to oxidative stress (TRPM2, (13)), high temperature (TRPM3, (14) as well as low temperature and cooling ligands such as menthol (TRPM8, (15)). Here, we coalesce ligand- and target-based approaches together with computational modelling to characterize how PZQ engages Sm.TRPMPZQ, with definition of a specific PZQ binding site revealing the molecular basis for the insensitivity of Fasciola to PZQ.

To characterize the pharmacological specificity of the schistosome TRP channel activated by PZQ (Sm.TRPMPZQ), we synthesized a series of 43 PZQ derivatives including several non-obvious analogues (Fig. S1, Table S1 and Supplementary Methods). The majority of analogues were structurally related to PZQ and the series included both resolved enantiomers ( $R$ )-PZQ (analogue 1) and (S)-PZQ (analogue 2), the major trans-(R)-4-OH PZQ metabolite (analogue $\mathbf{2 1},(9)$ ) and the veterinary drug epsiprantel (analogue 35, Fig. 1A). Following transient expression of Sm.TRPMPzQ in HEK293 cells, full concentration-response curves were performed for PZQ and each analogue, using a fluorescent assay to measure changes in intracellular $\mathrm{Ca}^{2+}$ (Fig. 1B and S2A). (R)-PZQ evoked a cytoplasmic $\mathrm{Ca}^{2+}$ signal with an $\mathrm{EC}_{50}$ of $0.46 \pm 0.12 \mu \mathrm{M}$ (Table S1). The enantiomer (S)-PZQ was $\sim 50$ fold less effective $\left(\mathrm{EC}_{50}=24.7 \pm 1.3 \mu \mathrm{M}\right)$, consistent with the activity ratio of enantiomers seen in vivo (9). The entire $\mathrm{EC}_{50}$ dataset for these analogues at Sm.TRPMPzQ (Fig. S2A, Table S1) was graphically represented as a color-coded heatmap (Fig. 1C). The majority of analogues displayed either no activity (19 compounds) or lower potency (13 compounds, $\mathrm{EC}_{50}$ values between 1-10 $\mu \mathrm{M} ; 7$ compounds, $\mathrm{EC}_{50}$ values $\geq 10 \mu \mathrm{M}$; Fig. 1C). 
A<smiles>O=C(C1CCCCC1)N1CC(=O)N2CCc3ccccc3[C@H]2C1</smiles><smiles>O=C(C1CCCCC1)N1CC(=O)N2CCc3ccccc3[C@H]2C1</smiles><smiles>C/C=C\C</smiles><smiles>O=C(C1CCCCC1)N1CC(=O)N2CCCc3ccccc3C2C1</smiles>

(R)-Praziquantel, 1

(S)-Praziquantel, 2

Metabolite, 21

Epsiprantel, $\mathbf{3 5}$

B

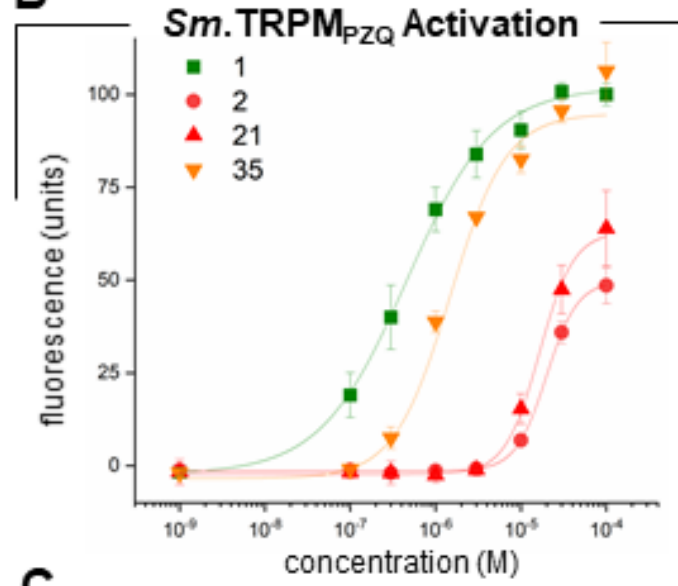

C

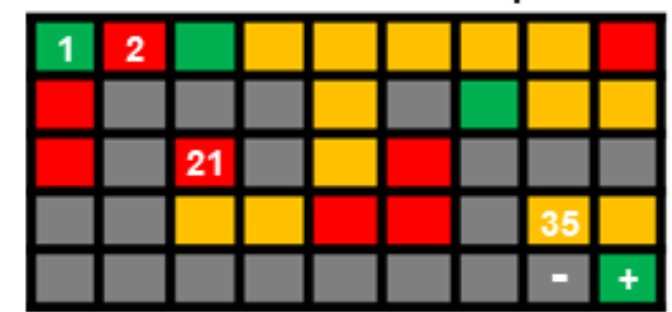

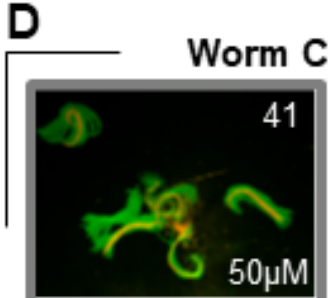
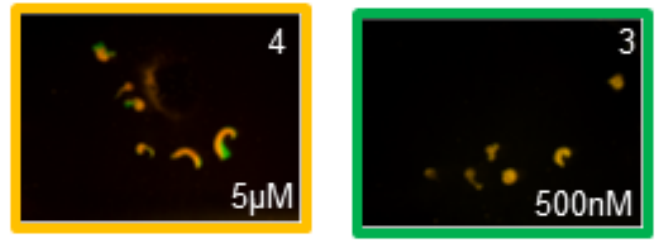

E

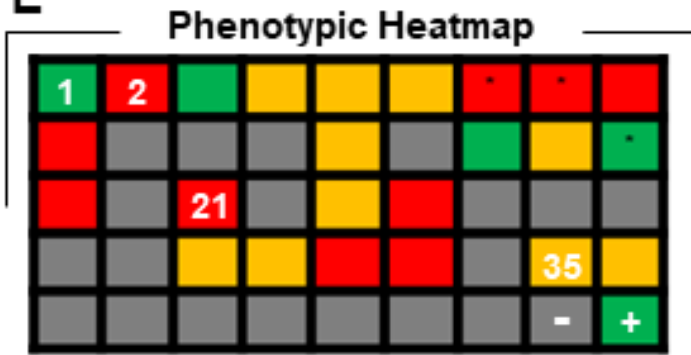

Fig. 1. Structure-activity relationships of $P Z Q$ analogues. (A) Chemical structure of key compounds: $(R)$-PZQ (analogue \#1), (S)-PZQ (analogue \#2), the major metabolite (trans-( $R$ )-4-OH PZQ, analogue \#21), and epsiprantel (analogue \#35) from the analogue collection fully indexed in Table S1. Colored circles highlight structural differences between these key analogues. (B) Concentration-response analysis for ( $R$ )-PZQ (analogue \#1), (S)-PZQ (analogue \#2), the major metabolite (trans-( $R$ )-4-OH PZQ, analogue \#21), and epsiprantel (analogue \#35) on Sm.TRPMPzo assessed by peak fluo-4 fluorescence. Data from $\geq 3$ independent experiments. (C) Graphical heatmap of the effects of each of the 43 analogues on Sm.TRPMPzQ activation. Each analogue is numbered left to right (1 through 43 ) in the heatmap and indexed to Table S1, with responses to vehicle (-) and PZQ (+) indicated (bottom right). Colors represent $\mathrm{EC}_{50}$ values $<1 \mu \mathrm{M}$ (green), $1-10 \mu \mathrm{M}$ (yellow), $>10 \mu \mathrm{M}$ (red) or inactive (grey). (D) Images of adult schistosome worms with single frame image (red) overlaid with maximum intensity projection of a time-lapse series to illustrate effect of analogues on worm motion 5 mins after treatment with analogues 41 (top left), 19 (top right), 4 (bottom right) and 3 (bottom left) at indicated concentrations. Strong contractile responses are shown at different concentrations, or inactivity (analog 41), as coded in the heatmap in (E). Scalebar, 1cm. (E) Graphical heatmap of the effects of each of the 43 analogues on worm contraction ordered as per (C). Differences between the two heatmaps are asterisked. 
As the relationship between target ( $S m$.TRPMPzQ) activation and worm phenotypic responses is unknown, we examined the ability of these same analogues to contract adult S. mansoni worms. Worm movement ex vivo was monitored before and after analogue addition. In the absence of drug exposure, movement of individual worms (red color) was evident by the area swept through by the worm in a maximal intensity projection of the recording (green color, Fig. 1D). The acute action of four different analogues (analogues 41, 19, 4 \& 3; Table S1) is shown in Figure 1D. These four analogues provide examples of dose-dependent inhibition of mobility over different concentrations ranges, or a lack of activity. An average motility score was calculated for each analogue relative to vehicle- and PZQ-treated worms (Fig. S2B) and used to produce a color-coded heatmap (Fig. 1E). Both the target (Sm.TRPMPzo) and phenotypic (worm contraction) heatmaps showed similar structure-activity relationships (Fig. 1C and 1E), confirming the pharmacological fingerprint of Sm.TRPMPZQ closely mirrors the activity of the same analogues at causing worm paralysis.

The structure-activity relationships (SAR) of these analogues revealed several trends. First, the cyclohexyl moiety in PZQ is a critical determinant for efficacy. Major modifications of this moiety yielded inactive or low potency analogs. Bulkier or more polar groups led to decreased potency with only minor alterations preserving comparable potency with (R)-PZQ (analogues 3 and 16). Second, modifications of the three-ringed PZQ core (core structure ' $A$ ', Fig. S1) were poorly tolerated with exception of a fluorinated scaffold (core structure ' $B$ ', analogues $30 \& 31$ ) or the enlarged piperidine ring of epsiprantel (Fig. 1A, analogues 35 \& 36). Third, stereochemically, all dextro- derivatives with the exception of (S)-PZQ were inactive. A ligand-based activity model confirmed the importance of these structural features (Fig. S2C), evidencing a 'tight' SAR with only small structural modifications of PZQ preserving activity at Sm.TRPMPZQ.

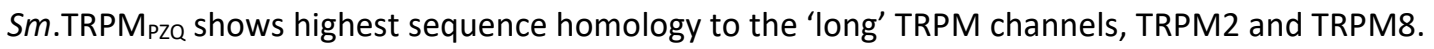
The presence of a $\mathrm{COOH}$ terminal NUDT9H-enzyme like domain in Sm.TRPMPzQ (Fig. 2A), diagnostic 
of TRPM2, may suggest a TRPM2-like activation mechanism. TRPM2 channels bind the channel activator ADP ribose at sites within the cytoplasmic regions in either the $\mathrm{COOH}$-terminus (NUDT9H domain) and/or the $\mathrm{NH}_{2}$-terminus (TRPM homology region, MHR 1/2domain (13, 16-19). Truncation of the C-terminal NUDT9H domain of Sm.TRPMPZQ did not impact responsivity to PZQ (Fig. 2A and S3A). $N$-terminal truncations into the MHR1/2 domains showed poor expression, consistent with the role of these domains in channel assembly and cell surface trafficking (20). However a panel of $N$ terminal point mutants targeting residues involved in ADP-ribose binding identified in other TRPM2 structures $(13,17)$ failed to prevent PZQ activation of Sm.TRPMPzQ (Table S2).

Structures of human TRPM8 ( $h$ TRPM8) reveal that the transmembrane region (voltage sensor-like domain (VSLD), transmembrane helices S1-S4), encompasses a conformationally malleable binding pocket capable of accommodating diverse chemotypes $(15,21)$. This is relevant as $(S)$-PZQ has been shown to activate human TRPM8 ( $h$ TRPM8), albeit at a high micromolar range (EC $\left.5_{50} \sim 20 \mu \mathrm{M}(22)\right)$. Using $h$ TRPM 8 structures as a template, point mutants of 11 known $h$ TRMP8 pocket-lining residues were generated. These mutants impaired $h$ TRPM 8 activation in response to various ligands (Fig. S4). Overall, the pattern of $h$ TRPM8 responsiveness to (S)-PZQ across this panel of $h$ TRPM8 mutants most closely resembled responses with the menthol-related cooling agent WS-12. These data suggest (S)-PZQ activates $h$ TRPM8 by interaction with the canonical $h$ TRPM8 transmembrane ligand binding pocket. Does an equivalent PZQ binding pocket exist in the transmembrane region of Sm.TRPM PzQ? Five residues $\left(\operatorname{Arg}^{1681}, \mathrm{Asn}^{1388}, \mathrm{Tyr}^{1392}, \mathrm{Arg}^{1514} \mathrm{Tyr}^{1678}\right.$ ) identical to residues lining the hTRPM8 binding pocket were mutated within Sm.TRPMPzQ (Fig. 2A). Four of these five mutants inhibited PZQ-evoked activation of Sm.TRPMPzQ (Fig. 2A and S3B). In contrast, neighboring point mutations within the same regions did not prevent PZQ-evoked Sm.TRPMPZQ activation (Table S4). These data suggest the existence of a TRPM8-like ligand binding site within the transmembrane region of Sm.TRPMPZQ. 


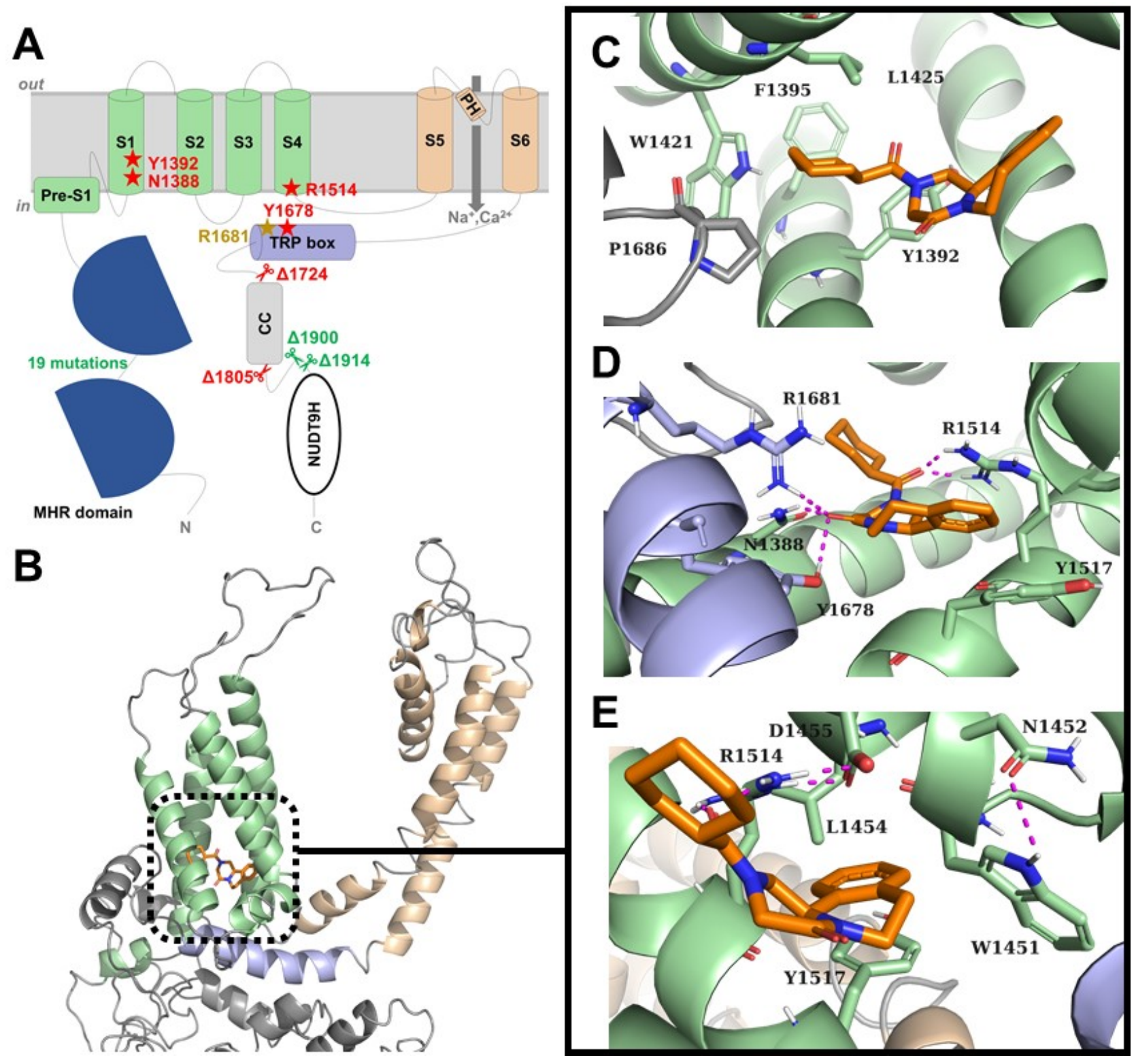

Fig. 2. Modeling the PZQ binding pocket in Sm.TRPMPzQ. (A) Schematic of domain organization of Sm.TRPMPZQ to highlight MHR domain, transmembrane spanning region (voltage-sensor-like domain, green; pore helices (PH), orange), TRP domain (blue), coiled-coil regions (CC) and $\mathrm{COOH}$ terminal NUDT9H domain. Truncation mutant (scissors) and specific point mutations (stars) analyzed in Figures S3A and S3B are highlighted. A total of 19 MHR domain point mutations (Table S2) did not affect $S m$.TRPM $M_{\text {PZ }}$ responsiveness to PZQ. Truncation mutants at the $\mathrm{COOH}$ terminus are indicated by their location and functional effect on PZQ activation (red, inhibitory; green, no effect). (B) Homology model of Sm.TRPMPZQ including docking pose of PZQ into the predicted binding site within the voltage-sensing ligand domain (VSLD, green) Pore domain (yellow) and TRP box domain (blue). (C) Hydrophobic cavity around cyclohexyl ring of PZQ. (D) PZQ and its non-covalent hydrogen bonding interactions (magenta) with binding site residues. (E) Hydrophobic cavity around aromatic ring of $P Z Q$ including ligand-sidechain and intra-residue hydrogen bond interactions (magenta). Single-letter code for amino acid residues: A, Ala; C, Cys; D, Asp; E, Glu; F, Phe; G, Gly; H, His; I, lle; K, Lys; L, Leu; M, Met; N, Asn; P, Pro; Q, Gln; R, Arg; S, Ser; T, Thr; V, Val; W, Trp; and Y, Tyr. 
Molecular modelling was employed to characterize the PZQ binding site. A homology model of the entire transmembrane region of Sm.TRPMPzo was built based from three known TRPM structures (Fig. 2B). Independent prediction tools identified a common binding site within the VSLD (Movie S1), in close proximity to the known $h$ TRPM8 transmembrane pocket (Fig. S5). Docking simulations with $(R)$-PZQ revealed PZQ bridged two hydrophobic cavities separated by a region of polar contacts. The cyclohexyl ring of PZQ projected into one hydrophobic cleft defined at its inner apex by three hydrophobic residues - Phe ${ }^{1395}(\mathrm{~S} 1), \operatorname{Trp}^{1421}(\mathrm{~S} 2)$ and $\mathrm{Leu}^{1425}(\mathrm{~S} 2)$ - with residues $\operatorname{Tyr}^{1392}$ (S1) and Pro $^{1686}$ (TRP) situated more peripherally around this ring (Fig. 2C). Within the polar region, the cyclohexyl carbonyl of PZQ forms a hydrogen bond with $\operatorname{Arg}^{1514}$ (S4-S5 linker) and the scaffold carbonyl of PZQ interacts with $\mathrm{Asn}^{1388}, \mathrm{Tyr}^{1678}$ and $\mathrm{Arg}^{1681}$ via hydrogen bonding (Fig. 2D). The second hydrophobic cavity accommodates the aromatic ring system of PZQ and is framed by six residues, $\operatorname{Trp}^{1451}$, Asn $^{1452}$, Leu ${ }^{1454}$, Asp $^{1455}$, Arg $^{1514}$ and Tyr ${ }^{1517}$ (Fig. 2E). A salt bridge between Asp ${ }^{1455}$ and $\operatorname{Arg}^{1514}$ shaped this cavity, in which the unsaturated part of the PZQ scaffold interacted with Tyr ${ }^{1517}$ (S4-S5 linker) via $\pi-\pi$ stacking and with $\operatorname{Arg}^{1514}$ (S4-S5 linker) via cation- $\pi$ interactions.

The impact of alanine mutagenesis of all 23 pocket-lining residues lying within a $5 \AA$ radius of the binding pose was examined (Fig. 3A and Table S3). Mutation of 20 of these 23 residues decreased PZQ sensitivity (Fig. S6A and S6B), with many point mutants displaying a complete loss of PZQevoked activity (Table S3). In contrast, alanine mutagenesis of an additional 26 amino acid residues localized away from the predicted binding pocket, minimally impacted PZQ activation of Sm.TRPMPZQ (Fig. S6C and Table S4). The localization of all 49 residues relative to the binding pocket is summarized in Figure S6. 


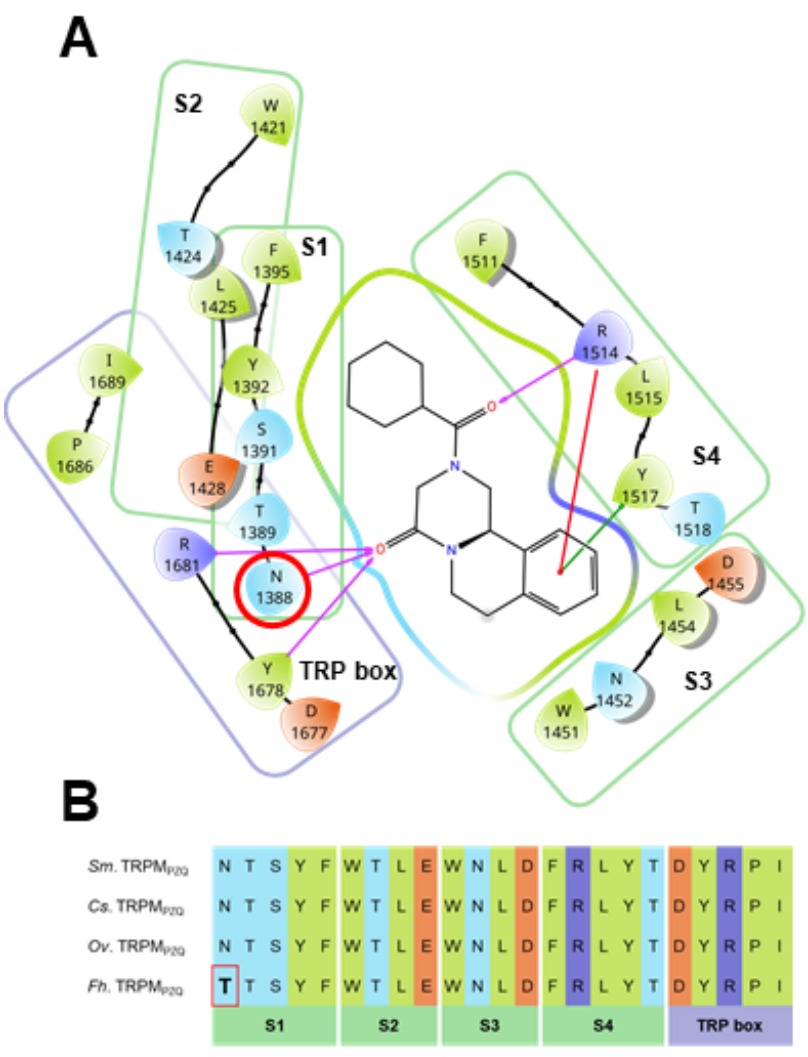

E Fh.TRPM $\mathrm{PZQ}_{\mathrm{O}}$
C
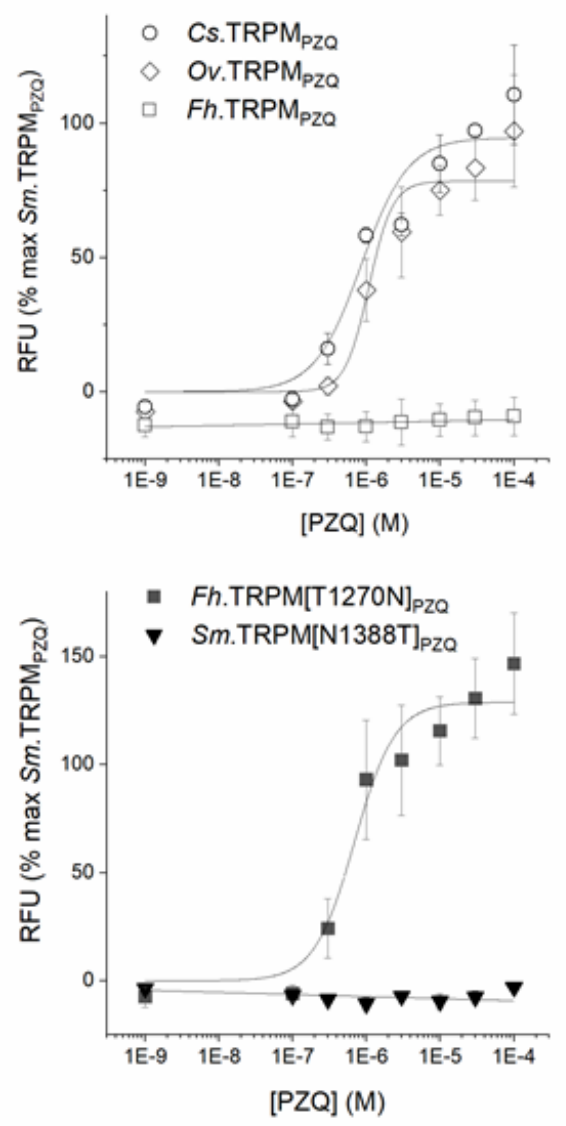

G
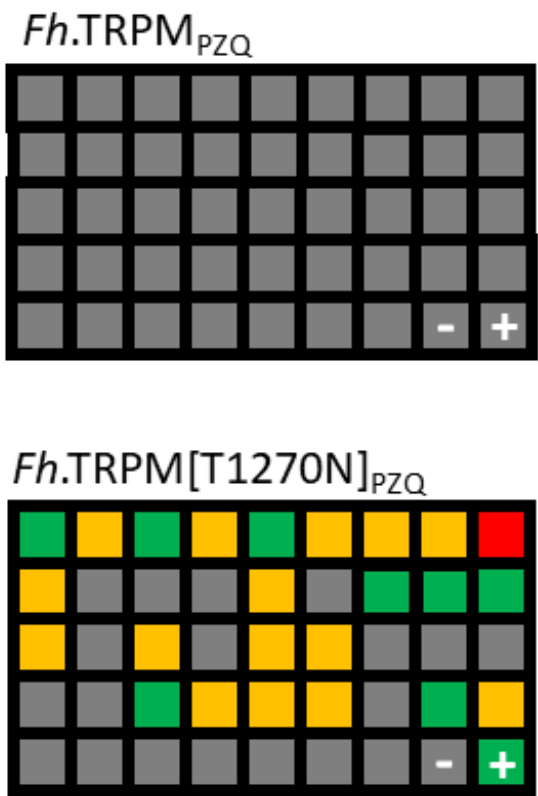

Fig. 3. Analysis of PZQ binding pocket in trematode TRPMPzQ channels. (A) Ligand-Receptor interaction map of $(R)$-PZQ with Sm.TRPMPZO showing amino acid residues within a $5 \AA$ radius and colored by properties (yellow: hydrophobic, blue: polar, purple: positively charged, orange: negatively charged). Interactions are highlighted by arrows (purple: hydrogen bonding, red: cation- $\pi$, green: $\pi-\pi$ stacking). (B) Alignment of 23 binding pocket residues identified in Sm.TRPMPzQ with other trematode TRPMPzQ channels (Clonorchis sinensis, Cs.TRPMPza; Opisthorchis viverrini, 
Ov.TRPMPzo and Fasciola hepatica, Fh.TRPMPzQ). Sequence data is from genomic annotations (Cs.TRPMPZQ, GAA53128.1; Ov.TRPMPZQ, OON13534.1; Fh.TRPMPZQ, THD26109.1). (C) Concentration response relationship for TRPMPzo from different liver flukes. (D) Concentration response curves for reciprocal binding pocket mutants of Schistosoma mansoni and Fasciola TRPMPzQ. (E \& F) Current traces recorded at $+60 \mathrm{mV}$ in HEK293 cells co-transfected with (E) Fh. TRPMPZQ and GFP, or (F) Fh.TRPM PZQ (Thr ${ }^{1270} \rightarrow$ Asn) and GFP, in the cell-attached configuration after addition of $0.1 \%$ DMSO (top, control) and $10 \mu \mathrm{M}$ PZQ (bottom). c, closed state. (G \& H) Graphical heatmap of the effects of each of the 43 analogues on (G) Fh.TRPMPzQ and (H) Fh.TRPM[T1270N] PzQ activation. Each analogue is numbered left to right (1 through 43) in the heatmap and indexed to Table S6, with responses to vehicle (-) and PZQ (+) indicated (bottom right). Grid is color-coded as Figure $1 \mathrm{C}$, with colors describing analogs with $\mathrm{EC}_{50} \mathrm{~S}<1 \mu \mathrm{M}$ (green), 1-10 $\mu \mathrm{M}$ (yellow), $>10 \mu \mathrm{M}$ (red) or inactive (grey).

This mutational analysis verified key aspects of the binding model. First, mutagenesis of channel residues predicted to interact with the carbonyl groups of PZQ (Fig. 2D and $3 \mathrm{~A}$ ) either caused a complete loss (Asn ${ }^{1388} \rightarrow$ Ala, $\operatorname{Arg}^{1514} \rightarrow$ Ala, Tyr $^{1678} \rightarrow$ Ala) or significant reduction $\left(\operatorname{Arg}^{1681} \rightarrow\right.$ Ala) in

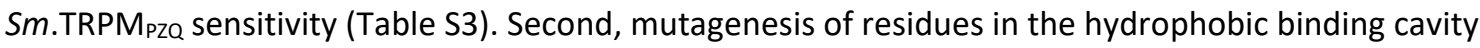
surrounding the cyclohexyl ring of PZQ led to complete loss of PZQ-evoked activity (Fig. 2C and Table S3). This hydrophobic cavity is tightly framed by residues from the S1 $\left(\operatorname{Tyr}^{392}\right.$, Phe $\left.{ }^{1395}\right)$ and S2 helices $\left(\operatorname{Trp}^{1421}\right.$, Leu $^{1425}$ ) as well as the TRP domain (Pro ${ }^{1686}$, Fig. 2C). Mutation of Leu ${ }^{1425}$, predicted to lie above the cyclohexyl ring of PZQ (Fig. 2C) to either bulkier (Leu ${ }^{1425} \rightarrow$ Phe) or smaller residues $\left(\right.$ Leu $^{1425} \rightarrow$ Ala) decreased PZQ sensitivity (Table S3). This is consistent with the analogue SAR analysis defining the cyclohexyl ring of PZQ as a key part of the pharmacophore and poorly tolerable to substitution. This requirement for activity conflicts with the incorporation of a polar hydroxyl group onto the cyclohexyl ring during phase 1 metabolism of PZQ (Fig. 1A and S2E). The major trans-(R)-4OH PZQ metabolite (analog 21, Fig. 1A), exhibits a 36-fold reduction in activity at Sm.TRPMPzQ (Tables S1 \& S5). Efforts to design more potent and metabolically-stable analogs to allow for lower dosing and sustained exposure may prove difficult in light of these observed structural requirements. Third, mutagenesis of residues framing the second hydrophobic cavity around the aromatic ring of PZQ led to complete loss $\left(\operatorname{Trp}^{1451}, \operatorname{Arg}^{1514}, \operatorname{Tyr}^{1517}\right.$ ) or decreased PZQ-evoked activity $\left(\right.$ Leu $^{1454}, \mathrm{Asn}^{1452}$; Table S3). Mutation of $\mathrm{Asp}^{1455} \rightarrow$ Ala, which electrostatically interacts with $\mathrm{Arg}^{1514}$ to define this pocket (Fig. 2E) also ablated PZQ sensitivity (Table S3). Interactions that orientate the (R)- 
PZQ cyclohexyl ring within the first hydrophobic pocket serve to preserve optimal $\pi-\pi$ stacking of the aromatic ring with $\operatorname{Tyr}^{1517}$ (S4) in this second hydrophobic pocket. (R)-PZQ therefore bridges two hydrophobic cavities, occupied by opposite ends of the PZQ molecule, to cause potent channel activation. For (S)-PZQ, the binding orientation is not optimal (Fig. S2D) providing a structural basis for the $\sim 50$-fold difference in $\mathrm{EC}_{50} \mathrm{~S}$ of the enantiomers at $\mathrm{Sm}$.TRPMPzQ. Even subtle changes in structure as seen with the veterinary drug epsiprantel (Fig. 1A, analog 35), which contains a sevenmembered azepine ring, alter ligand conformation (Fig. S2F) and result in lower potency. Therefore, both modeling and mutagenesis data converge to establish that (R)-PZQ engages Sm.TRPMPZQ via a transmembrane binding pocket to cause channel activity, $\mathrm{Ca}^{2+}$ influx and worm contraction.

PZQ is used to treat other parasitic flatworm infections, such as clonorchiasis and opisthorchiasis (23). The sequences of the TRPM homologs in the liver flukes Clonorchis sinensis and Opisthorchis viverrini displayed complete conservation of the 23 residues that most closely circumscribe the PZQ binding site (Fig. 3B). Heterologous expression of Cs.TRPMPZQ and Ov.TRPMPzQ conferred PZQ-evoked $\mathrm{Ca}^{2+}$ signals in transfected HEK293 cells. The observed sensitivities of the fluke TRPMPzQ channels to PZQ (Cs.TRPMPZQ, EC $\mathrm{E}_{50}=0.85 \pm 0.22 \mu \mathrm{M} ;$ Ov.TRPMPZQ, $\mathrm{EC}_{50}=1.07 \pm 0.33 \mu \mathrm{M} ; \mathrm{Fig} .3 \mathrm{C}$ ) were similar to Sm.TRPM PZQ. One parasitic flatworm infection that is refractory to PZQ treatment is fascioliasis, a disease of considerable clinical and veterinary importance worldwide. The Fasciola hepatica TRPMPZQ channel (Fh.TRPMPZQ) did not respond to PZQ (Fig. 3C), unlike the TRPMPZQ channels in these other PZQ-sensitive flukes. Sequence analysis of Fh.TRPMPzQ revealed a single amino acid difference in the predicted PZQ binding pocket compared with PZQ-sensitive TRPMPZQ channels where a threonine residue in $\mathrm{S} 1$ of Fh.TRPMPZQ replaced an asparagine residue present in the other homologs (Fig. 3B). This difference reflects a single nucleotide difference in the genomic sequence of these flatworms ('ACT' encoding threonine in Fasciola, 'AAT' encoding asparagine in Schistosoma). The resulting asparagine residue in the PZQ-sensitive channels is predicted to be a critical residue that interacts with the core carbonyl of PZQ (Fig. 2D and 3A). 
To test the impact of substitution at this residue, reciprocal point mutants were generated in Sm.TRPMPZQ $\left(\right.$ Asn $\left.^{1388} \rightarrow \operatorname{Thr}\right)$ and Fh.TRPMPZQ $\left(\operatorname{Thr}^{1270} \rightarrow\right.$ Asn). The asparagine to threonine mutation in the schistosome channel caused a loss of PZQ-evoked activity (Fig. 3D). Reciprocally, the threonine to asparagine mutation in the Fasciola channel ( $\operatorname{Thr}^{1270} \rightarrow$ Asn) caused a gain of responsiveness to PZQ (Fig. 3D), with the mutant Fasciola channel being robustly activated by PZQ ( $\left.E C_{50}=0.55 \pm 0.1 \mu \mathrm{M}\right)$. The properties of the Fasciola channels were then assessed electrophysiologically. Whereas PZQ

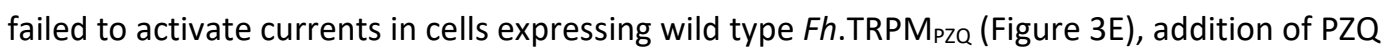

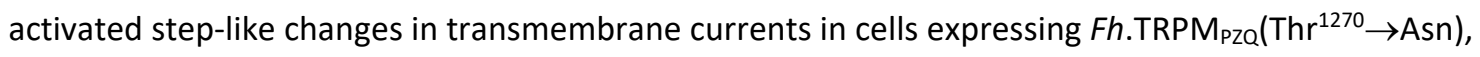
consistent with PZQ-evoked single channel activity (Figure 3F). Therefore, two orthogonal assays $\left(\mathrm{Ca}^{2+}\right.$ imaging and electrophysiology) demonstrate a single point mutation in the Fasciola binding pocket results in a gain of sensitivity to PZQ. These data reveal a molecular basis for the natural insensitivity of Fasciola to PZQ chemotherapy.

Finally, we screened the 43 analogues used to define structure-activity relationships at Sm.TRPMPzQ

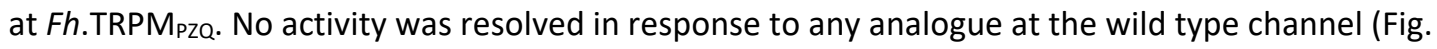
3G). In contrast, in cells expressing Fh.TRPMPzQ(Thr ${ }^{1270} \rightarrow$ Asn), several analogues displayed activity (Fig. 3H). The structure-activity pattern was identical at both schistosome and liver fluke channels (compare Fig. $1 \mathrm{C}$ with Fig $3 \mathrm{H}$ ), with the same 24 analogs that activated Sm.TRPMPZQ also activating Fh.TRPMPzQ $\left(\operatorname{Thr}^{1270} \rightarrow\right.$ Asn). Fh.TRPMPzQ $\left(\operatorname{Thr}^{1270} \rightarrow\right.$ Asn $)$ displayed $\sim 3$-fold higher sensitivity to the active analogs, consistent with a higher sensitivity of Fh.TRPMPzQ $\left(\operatorname{Thr}^{1270} \rightarrow\right.$ Asn) to PZQ compared with Sm.TRPMPZQ.

In conclusion, this study identifies a binding site for $(R)$-PZQ in a juxta-membrane cavity within the VSLD domain of TRPMPZQ, a broadly conserved parasitic flatworm ion channel. Identification of a hTRPM8-like binding pocket within an invertebrate TRPM2-like channel architecture was surprising, 
but highlighting how little we know about the properties of flatworm TRPs (24) which have specialized along evolutionary trajectories distinct from human TRP channels. Mutagenesis of the PZQ binding site in Sm.TRPMPZQ demonstrates that single point mutations ablate responsiveness to PZQ, and the properties of wild-type Fh.TRPMPZQ evidence a naturally occurring TRPMPZQ channel that is insensitive to PZQ. The significance of these changes in context of reports of decreased clinical effectiveness of PZQ in the field $(25,26)$, or the lower sensitivity of isolated schistosome strains to PZQ $(27,28)$, is important to monitor given the critical need to preserve the effectiveness of PZQ for treating this burdensome neglected tropical disease. Definition of the $(R)$-PZQ binding site in this TRP channel now provides impetus to design novel therapies that exploit the druggability of TRPMPZQ in various disease-causing parasites.

\section{References and Notes}

1. D. G. Colley, A. L. Bustinduy, W. E. Secor, C. H. King, Human schistosomiasis. Lancet 383, 22532264 (2014).

2. C. H. King, M. Dangerfield-Cha, The unacknowledged impact of chronic schistosomiasis. Chronic IIIn 4, 65-79 (2008).

3. A. K. Deol et al., Schistosomiasis - Assessing Progress toward the 2020 and 2025 Global Goals. N. Engl. J. Med. 381, 2519-2528 (2019).

4. Ending the neglect to attain the Sustainable Development Goals - A road map for neglected tropical diseases 2021-2030. World Health Organ. Monogr. Ser., (2020).

5. P. M. Selzer, C. Epe, Antiparasitics in Animal Health: Quo Vadis? Trends Parasito/ 37, 77-89 (2021).

6. P. Andrews, H. Thomas, R. Pohlke, J. Seubert, Praziquantel. Med. Res. Rev. 3, 147-200 (1983).

7. R. Pax, J. L. Bennett, R. Fetterer, A benzodiazepine derivative and praziquantel: effects on musculature of Schistosoma mansoni and Schistosoma japonicum. Naunyn. Schmiedebergs Arch. Pharmacol. 304, 309-315 (1978).

8. U. Staudt, G. Schmahl, G. Blaschke, H. Mehlhorn, Light and scanning electron microscopy studies on the effects of the enantiomers of praziquantel and its main metabolite on Schistosoma mansoni in vitro. Parasitol. Res. 78, 392-397 (1992).

9. I. Meister et al., Activity of praziquantel enantiomers and main metabolites against Schistosoma mansoni. Antimicrob. Agents Chemother. 58, 5466-5472 (2014).

10. M. C. Jeziorski, R. M. Greenberg, Voltage-gated calcium channel subunits from platyhelminths: potential role in praziquantel action. Int. J. Parasitol. 36, 625-632 (2006).

11. C. M. Thomas, D. J. Timson, The Mechanism of Action of Praziquantel: Six Hypotheses. Curr Top Med Chem 18, 1575-1584 (2018).

12. S. K. Park et al., The anthelmintic drug praziquantel activates a schistosome transient receptor potential channel. J. Biol. Chem. 294, 18873-18880 (2019).

13. Y. Huang, P. A. Winkler, W. Sun, W. Lu, J. Du, Architecture of the TRPM2 channel and its activation mechanism by ADP-ribose and calcium. Nature 562, 145-149 (2018). 
14. J. Vriens et al., TRPM3 is a nociceptor channel involved in the detection of noxious heat. Neuron 70, 482-494 (2011).

15. M. M. Diver, Y. Cheng, D. Julius, Structural insights into TRPM8 inhibition and desensitization. Science 365, 1434-1440 (2019).

16. Z. Zhang, B. Toth, A. Szollosi, J. Chen, L. Csanady, Structure of a TRPM2 channel in complex with $\mathrm{Ca}(2+)$ explains unique gating regulation. eLife 7, 36409 (2018).

17. Y. Huang, B. Roth, W. Lu, J. Du, Ligand recognition and gating mechanism through three ligand-binding sites of human TRPM2 channel. eLife 8, (2019).

18. L. Wang et al., Structures and gating mechanism of human TRPM2. Science 362, (2018).

19. F. J. Kuhn, C. Kuhn, M. Winking, D. C. Hoffmann, A. Luckhoff, ADP-Ribose Activates the TRPM2 Channel from the Sea Anemone Nematostella vectensis Independently of the NUDT9H Domain. PLoS One 11, e0158060 (2016).

20. C. B. Phelps, R. Gaudet, The role of the $\mathrm{N}$ terminus and transmembrane domain of TRPM 8 in channel localization and tetramerization. J. Biol. Chem. 282, 36474-36480 (2007).

21. Y. Yin et al., Structural basis of cooling agent and lipid sensing by the cold-activated TRPM8 channel. Science 363, (2019).

22. G. S. Gunaratne, N. A. Yahya, P. I. Dosa, J. S. Marchant, Activation of host transient receptor potential (TRP) channels by praziquantel stereoisomers. PLoS Negl Trop Dis 12, e0006420 (2018).

23. J. Y. Chai, Praziquantel treatment in trematode and cestode infections: an update. Infect Chemother 45, 32-43 (2013).

24. S. Bais, R. M. Greenberg, TRP channels as potential targets for antischistosomals. International Journal for Parasitology. Drugs and Drug Resistance 8, 511-517 (2018).

25. M. Ismail et al., Characterization of isolates of Schistosoma mansoni from Egyptian villagers that tolerate high doses of praziquantel. Am. J. Trop. Med. Hyg. 55, 214-218 (1996).

26. S. William, S. Botros, Validation of sensitivity to praziquantel using Schistosoma mansoni worm muscle tension and $\mathrm{Ca}^{2+}$-uptake as possible in vitro correlates to in vivo $\mathrm{ED}_{50}$ determination. Int. J. Parasitol. 34, 971-977 (2004).

27. P. G. Fallon, M. J. Doenhoff, Drug-resistant schistosomiasis: resistance to praziquantel and oxamniquine induced in Schistosoma mansoni in mice is drug specific. Am. J. Trop. Med. Hyg. 53, 61-62 (1995).

28. F. F. Couto et al., Schistosoma mansoni: a method for inducing resistance to praziquantel using infected Biomphalaria glabrata snails. Mem. Inst. Oswaldo Cruz 106, 153-157 (2011).

29. T. Cheeseright, M. Mackey, S. Rose, A. Vinter, Molecular field extrema as descriptors of biological activity: definition and validation. J Chem Inf Model 46, 665-676 (2006).

30. G. M. Sastry, M. Adzhigirey, T. Day, R. Annabhimoju, W. Sherman, Protein and ligand preparation: parameters, protocols, and influence on virtual screening enrichments. $J$. Comput. Aided Mol. Des. 27, 221-234 (2013).

31. T. A. Halgren, Identifying and characterizing binding sites and assessing druggability. J Chem Inf Model 49, 377-389 (2009).

32. A. Volkamer, D. Kuhn, T. Grombacher, F. Rippmann, M. Rarey, Combining global and local measures for structure-based druggability predictions. J Chem Inf Model 52, 360-372 (2012).

33. Concert-Pharmaceuticals-Inc., Pyrazinoisoquinoline compounds as antiparasitic agents. Patent WO 2012122028.

34. Y. Dong et al., Praziquantel analogs with activity against juvenile Schistosoma mansoni. Bioorg. Med. Chem. Lett. 20, 2481-2484 (2010).

35. M. Ahamed, B. Chan, P. Jensen, M. H. Todd, The outcome of the oxidations of unusual enediamide motifs is governed by the stabilities of the intermediate iminium ions. PLoS One 7, e47224 (2012).

36. M. D. Brewer et al., Synthesis and anthelmintic activity of a series of pyrazino[2,1a][2]benzazepine derivatives. J. Med. Chem. 32, 2058-2062 (1989). 
37. P. S. Sadhu et al., Synthesis of new praziquantel analogues: potential candidates for the treatment of schistosomiasis. Bioorg. Med. Chem. Lett. 22, 1103-1106 (2012).

Acknowledgements: Funding: This work was supported by NIH R01-AI145871 (JSM), NIH F31Al145091 (NAY) and the Marcus Family. Schistosome-infected mice were provided by the NIAID Schistosomiasis Resource Center at the Biomedical Research Institute (Rockville, MD) through NIHNIAID Contract HHSN272201000005I for distribution via BEI Resources. Non-Author Contributions: DM acknowledges Dr. Andreas Waechtler, Jeremy Maurin, Mathilde Dreyer, Philipp Rietsch, Marie Emering, Fabian Gramain and Minas Stefanou for their contributions to chemical synthesis. Author Contributions: Experiments were performed by SKP, NY, CR. Chemical synthesis of PZQ analogues was directed by DM. Computational chemistry and molecular modeling were performed by LF and FR. Structure-activity relationship studies were directed by TS. SKP, LF, TS and JSM designed experimental analyses, TS and JSM wrote the paper with help from all authors. Competing interests: LF, DM and FR are employees of Merck KGaA. TS is an employee of Ares Trading SA, an affiliate of Merck KGaA, Darmstadt, Germany. All data are available in the main text or supplementary materials.

\section{Supplementary Materials:}

Materials and Methods

Figs. S1 to S6

Tables S1 to S5

Movie S1 